\title{
ON ASYMPTOTIC BEHAVIOR OF THE MASS OF RAYS
}

\author{
TAKASHI SHIOYA
}

(Communicated by Jonathan M. Rosenberg)

\begin{abstract}
We consider the measure of the set of all unit vectors tangent to rays emanating from a point $p$ in a finitely connected complete open Riemannian 2-manifold $M$. If $M$ with one end admits total curvature $c(M)$, then this measure tends to $\min \{2 \pi \chi(M)-c(M), 2 \pi\}$ as $p$ tends to infinity, where $\chi(M)$ is the Euler characteristic.
\end{abstract}

\section{INTRODUCTION}

Let $M$ be a complete, noncompact, connected, oriented and finitely connected Riemannian 2-manifold without boundary. The total curvature $c(M)$ of $M$ is defined by the improper integral over $M$ of Gaussian curvature $G$ :

$$
c(M):=\int_{M} G d M,
$$

where $d M$ is the area element of $M$. It is a well-known theorem due to CohnVossen [1] that if $M$ admits total curvature, then $c(M) \leq 2 \pi \chi(M)$, where $\chi(M)$ is the Euler characteristic of $M$. We study asymptotic behavior of the mass of rays in terms of the total curvature of a complete open surface.

A ray $\gamma:[0, \infty) \rightarrow M$ is defined to be a geodesic any subarc of which is a minimizing segment joining its endpoints. We denote the tangent space of $M$ at $p$ by $M_{p}$. Let $S_{p} \subset M_{p}$ be the set of all unit vectors at $p$ and let $A_{p} \subset S_{p}$ be the set of all unit vectors tangent to rays emanating from $p$. We denote by "meas" the Lebesgue measure on the unit circle $S_{p}$ with the total measure $2 \pi$. Since the limit of a sequence of rays in $M$ is a ray, $A_{p}$ is a closed and measurable subset of $S_{p}$ and the function $p \mapsto \operatorname{meas}\left(A_{p}\right)$ is uppersemicontinous. Thus this function is locally integrable in the sense of Lebesgue.

The first result on the relation between the total curvature and the measure of $A_{p}$ was obtained by Maeda.

Received by the editors December 30, 1988 and, in revised form, March 3, 1989.

1980 Mathematics Subject Classification (1985 Revision). Primary 53C20.

Key words and phrases. Complete open manifolds, Gauss-Bonnet theorem, geodesics, rays, total curvature. 
Theorem (Maeda [6]). If $M$ is homeomorphic to $R^{2}$ and if it has nonnegative Gaussian curvature everywhere, then

$$
\inf _{p \in M} \operatorname{meas}\left(A_{p}\right)=2 \pi-c(M) .
$$

In [10], Shiga extended this result to the case when the sign of Gaussian curvature changes. Moreover, Oguchi [8] extended their results to the case when $M$ has only one end. Shiohama proved the following integral formula for the mass of rays.

Theorem (Shiohama [13]). Assume that $M$ with one end admits total curvature with $2 \pi \chi(M)-c(M)<2 \pi$. If $\left\{K_{j}\right\}$ is a monotone increasing sequence of compact sets with $\bigcup K_{j}=M$, then

$$
\lim _{j \rightarrow \infty} \frac{\int_{K_{j}} \operatorname{meas}\left(A_{p}\right) d M}{\int_{K_{j}} d M}=2 \pi \chi(M)-c(M) .
$$

The following Theorem A which will be proved in $\S 2$ plays an essential role throughout this paper.

Theorem A. Assume that $M$ with one end admits total curvature. Let $\left\{p_{j}\right\}$ be an arbitrary divergent sequence of points of $M$. Then,

$$
\lim _{j \rightarrow \infty} \operatorname{meas}\left(A_{p_{j}}\right)=\min \{2 \pi \chi(M)-c(M), 2 \pi\} .
$$

In the case when $2 \pi \chi(M)-c(M)<2 \pi$, Theorem A was proved by Shiohama in the proof of the integral formula $(*)$. A crucial point of the proof of $(*)$ in the case when $2 \pi \chi(M)-c(M)<2 \pi$ is nonexistence of straight lines. We emphasize that in our case $M$ admits straight lines and this situation makes the proof difficult. To overcome this difficulty we need delicate arguments as developed in Lemmas $2.1,2.2,2.3$, and 2.4. In $\S 2$, we will prove Theorem A in the case when $2 \pi \chi(M)-c(M) \geq 2 \pi$. We can extend Theorem A to the case when $M$ has more than one end as stated in Theorem B.

In $\S 3$, we will discuss the case when $M$ has finitely many ends. To state Theorem B some definitions and notations are needed. Assume that $M$ is finitely connected with $k$ ends and that $M$ admits total curvature. Let $K$ be a compact domain on $M$ such that $M-\operatorname{Int}(K)$ is a union of disjoint closed half cylinders $U_{1}, \ldots, U_{k}$ (we call them tubes) and $\partial K$ consists of $k$ simple closed piecewise smooth curves. For any domain $D$ bounded by piecewise smooth curves $c_{1}, \ldots, c_{n}$ each of which is parametrized positively by arc length relative to $D$, we denote by $\kappa(D)$ the sum of curvature integrals of $c_{1}, \ldots, c_{n}$ and of the outer angles at all the vertices of $D$. If we set $s_{i}(M):=-c\left(U_{i}\right)-\kappa\left(U_{i}\right)$ for $i=1, \ldots, k$, then

$$
\sum_{1 \leq i \leq k} s_{i}(M)=2 \pi \chi(M)-c(M) .
$$

The value $s_{i}(M)$ does not depend on the choice of tube $U_{i}$ by the Gauss-Bonnet Theorem. 
With these notations Theorem B is stated as follows.

Theorem B. Assume that $M$ with $k$ ends admits total curvature. Let $U_{i}$ be a tube of $M$ and let $\left\{p_{j}\right\}$ be an arbitrary divergent sequence of points of $U_{i}$. Then

$$
\lim _{j \rightarrow \infty} \operatorname{meas}\left(A_{p_{j}}\right)=\min \left\{s_{i}(M), 2 \pi\right\} .
$$

The following Corollaries C, D, and Theorem E are straightforward consequences of Theorem B and the isoperimetric inequality stated in Lemma 1.3 (2) (see [14]). The proofs are omitted here.

Corollary C. Under the same assumption as in Theorem B, let $\left\{p_{j}\right\}$ be an arbitrary divergent sequence of points of $M$. Then,

$$
\begin{aligned}
\min _{1 \leq i \leq k}\left\{s_{i}(M), 2 \pi\right\} & \leq \liminf _{j \rightarrow \infty} \operatorname{meas}\left(A_{p_{j}}\right) \\
& \leq \limsup _{j \rightarrow \infty} \operatorname{meas}\left(A_{p_{j}}\right) \\
& \leq \max _{1 \leq i \leq k} \min \left\{s_{i}(M), 2 \pi\right\} .
\end{aligned}
$$

Theorem D. Assume that $M$ with $k$ ends admits total curvature. Let $\left\{K_{j}\right\}$ be a monotone increasing sequence of compact subsets of $M$ with $\bigcup K_{j}=M$. Then we have

$$
\begin{aligned}
\min _{1 \leq i \leq k}\left\{s_{i}(M), 2 \pi\right\} & \leq \liminf _{j \rightarrow \infty} \frac{\int_{K_{j}} \operatorname{meas}\left(A_{p}\right) d M}{\int_{K_{j}} d M} \\
& \leq \limsup _{j \rightarrow \infty} \frac{\int_{K_{j}} \operatorname{meas}\left(A_{p}\right) d M}{\int_{K_{j}} d M} \\
& \leq \max _{1 \leq i \leq k} \min \left\{s_{i}(M), 2 \pi\right\} .
\end{aligned}
$$

Theorem E. Assume that $M$ with $k$ ends admits total curvature. Let $c$ be a simple closed smooth curve in $M$ and let $B(t):=\{x \in M ; d(x, c)<t\}$. Then we have

$$
\lim _{t \rightarrow \infty} \frac{\int_{B(t)} \operatorname{meas}\left(A_{p}\right) d M}{\int_{B(t)} d M}= \begin{cases}\frac{\sum_{1 \leq i \leq k} s_{i}(M) \min \left\{s_{i}(M), 2 \pi\right\}}{2 \pi \chi(M)-c(M)} & \text { if } 2 \pi \chi(M)-c(M)>0, \\ 0 & \text { if } 2 \pi \chi(M)-c(M)=0 .\end{cases}
$$

\section{Preliminaries}

In this section, we state the notations and lemmas used for the proof of our results. Let $M$ be a finitely connected complete open 2-manifold admitting total curvature. Let $D \subset M$ be a domain as stated in $\S 0$. Then $\kappa(D)$ has the following properties.

(1.1) $\kappa(D)=-\kappa(M-D)$.

(1.2) If $D$ is bounded, then $c(D)=2 \pi \chi(D)-\kappa(D)$.

(1.3) Assume that $\partial D$ consists of a curve $c$ homeomorphic to a line such that $c|(-\infty, a], c|[b, \infty)$ are geodesics for some $a, b \in R$. Then,

$$
c(D) \leq 2 \pi \chi(D)-\pi-\kappa(D) .
$$


(1.4) In (1.3), if $d_{D}(c(t), c(-t)) \geq 2 t-r$ for all $t \geq 0$ and for some constant $r \geq 0$, then

$$
c(D) \leq 2 \pi \chi(D)-2 \pi-\kappa(D)
$$

where $d_{D}$ is the inner distance on $\mathrm{Cl}(D)$, the closure of $D$, induced from the Riemannian structure of $M$.

(1.1) is obvious. (1.2) follows from the Gauss-Bonnet Theorem. (1.3) and (1.4) are due to Cohn-Vossen [2].

For the rest of this section we assume that $M$ has only one end. The following Lemma 1.1 plays an important role for the proof of Lemmas 2.1 and 2.2 .

Lemma 1.1 (Shiga [10]). Let $\sigma, \gamma$ be rays emanating from a point $p$ in $M$. Assume that $\sigma \cup \gamma$ bounds a domain $D$ and that $\operatorname{Int}(D)$ does not contain any ray emanating from $p$. If $\theta$ is the inner angle of $D$ at $p$, then we have

$$
c(D)=2 \pi \chi(D)-2 \pi+\theta .
$$

The proof of Lemma 1.1 proceeds in outline as follows. Since $\operatorname{Int}(D)$ does not contain any ray emanating from $p$, there is a divergent sequence $\left\{q_{j}\right\}$ in $D$ with the property that there exist two minimizing geodesics $\xi_{j}$ and $\eta_{j}$ joining $p$ to $q_{j}$ in $D$ such that $\lim \xi_{j}=\gamma$ and $\lim \eta_{j}=\sigma$ and such that the inner angle at $q_{j}$ of the disk domain $D_{j}$ in $D$ bounded by $\xi_{j}$ and $\eta_{j}$ tends to zero as $j \rightarrow \infty$. The sequence $\left\{D_{j}\right\}$ of disk domains is monotone increasing and satisfies $\bigcup D_{j}=D$.

For a point $p \in M$ and for $u \in A_{p}$ let $\gamma_{u}(t):=\exp _{p} t u$ for $t \geq 0$. Let $K$ be an arbitrary fixed compact domain bounded by a piecewise smooth closed curve such that $M-K$ is an open half cylinder. For any geodesic $\gamma$ passing through a point of $K$, set

$$
t_{0}(\gamma):=\min \{t ; \gamma(t) \cap \partial K\}, \quad t_{1}(\gamma):=\max \{t ; \gamma(t) \cap \partial K\} .
$$

For a point $p \in M-K$, set

$$
\begin{aligned}
& A_{p}(K):=\left\{v \in A_{p} ; \gamma_{v}([0, \infty)) \cap K \neq \varnothing\right\}, \\
& A_{p}^{\prime}(K):=\left\{v \in A_{p} ; \gamma_{v}([0, \infty)) \cap \operatorname{Int}(K)=\varnothing\right\} .
\end{aligned}
$$

For $u, v \in A_{p}(K)$, the two subarcs $\gamma_{u}\left(\left[0, t_{0}\left(\gamma_{u}\right)\right]\right), \gamma_{v}\left(\left[0, t_{0}\left(\gamma_{v}\right)\right]\right)$ of the rays $\gamma_{u}, \gamma_{v}$ and a subarc of $\partial K$ joining $\gamma_{u}\left(t_{0}\left(\gamma_{u}\right)\right)$ to $\gamma_{v}\left(t_{0}\left(\gamma_{v}\right)\right)$ together form a simple closed curve in $M-\operatorname{Int}(K)$ which bounds an open disk domain $\Delta_{p}(u, v)$ in $M-K$. If we set

$$
\Delta_{p}(K):=\bigcup_{u, v \in A_{p}(K)} \Delta_{p}(u, v)
$$

and if $\theta_{p}(K)$ is the inner angle of $\Delta_{p}(K)$ at $p$, then the following lemma is true.

Lemma $1.2([14])$. For any divergent sequence $\left\{p_{i}\right\}$ of points in $M-K, \theta_{p_{i}}(K)$ tends to zero as $i \rightarrow \infty$. 
The following isoperimetric inequality is used for the proof of our integral formulas. For a point $p \in M$ let

$$
S_{t}(p):=\{x \in M ; d(p, x)=t\}, \quad B_{t}(p):=\{x \in M ; d(p, x)<t\} .
$$

Lemma 1.3 (Hartman [4] and Shiohama [11]).

(1) There exists a constant $R>0$ such that for almost all $t \geq R, S_{t}(p)$ is a simple closed curve of class $C^{\infty}$ except finitely many cut points from $p$.

(2) We have

$$
\begin{aligned}
\lim _{t \rightarrow \infty} \frac{L\left(S_{t}(p)\right)}{t} & =\lim _{t \rightarrow \infty} \frac{2 \operatorname{Area}\left(B_{t}(p)\right)}{t^{2}}=\lim _{t \rightarrow \infty} \frac{L\left(S_{t}(p)\right)^{2}}{2 \operatorname{Area}\left(B_{t}(p)\right)} \\
& =2 \pi \chi(M)-c(M),
\end{aligned}
$$

where $L(\alpha)$ is the length of a curve $\alpha$.

\section{THE CASE WHEN $M$ HAS ONE END}

In this section, we assume that $M$ has only one end and that $M$ admits total curvature with $2 \pi \chi(M)-c(M) \geq 2 \pi$. Let $K$ be a compact domain such that $M-K$ is an open half cylinder and $p$ a point in $M-K$. Assume that $A_{p}^{\prime}(K)$ is nonempty. Then we denote by $D_{p}(K)$ the unique component of $M-\left\{\exp _{p} t v ; v \in A_{p}^{\prime}(K), t \geq 0\right\}$ such that $K \subset D_{p}(K)$.

Lemma 2.1. Let $\left\{p_{i}\right\}$ be a divergent sequence of points of $M$. Assume that for any compact set $K$ such that $M-K$ is an open half cylinder, $A_{p_{i}}^{\prime}(K)$ is nonempty for all sufficiently large $i$ and that the inner angle $\theta_{i}$ of $D_{p_{i}}(K)$ at $p_{i}$ tends to zero as $i \rightarrow \infty$. Then

$$
\lim _{i \rightarrow \infty} \operatorname{meas}\left(A_{p_{i}}\right)=2 \pi .
$$

Proof. Let $\varepsilon$ be an arbitrary given positive number. Let $K$ be a compact subset of $M$ such that $M-K$ is an open half cylinder and such that

$$
\int_{M-K} G^{+} d M<\varepsilon
$$

For each $i$, let $\left\{E_{i, j}\right\}_{j}$ be the family of all connected components of

$$
M-\left(\left\{\exp _{p} t v ; v \in A_{p_{i}}, t \geq 0\right\} \cup D_{p_{i}}(K)\right) .
$$

Each $E_{i, j}$ is an open half plane bounded by two rays emanating from $p_{i}$, and has the property that there are no rays emanating from $p_{i}$ in it. Lemma 1.1 implies that $c\left(E_{i, j}\right)$ is equal to the inner angle of $E_{i, j}$ at $p_{i}$. It follows from (2.1.1) that

$$
2 \pi-\operatorname{meas}\left(A_{p_{i}}\right)-\theta_{i}=c\left(\bigcup_{j} E_{i, j}\right)<\varepsilon
$$

for all $i$. Since $\theta_{i}$ tends to zero as $i \rightarrow \infty$ and $\varepsilon$ is arbitrary, this completes the proof. 
Lemma 2.2. Let $\left\{p_{i}\right\}$ be a divergent sequence of points of $M$. Assume that for any compact set $K$, there exists a point $p(K) \in\left\{p_{i}\right\}$ such that $A_{p(K)}(K)$ is empty. Then, there exists a subsequence $\left\{p_{j}\right\}$ of $\left\{p_{i}\right\}$ such that

$$
\lim _{j \rightarrow \infty} \operatorname{meas}\left(A_{p_{j}}\right)=2 \pi \text {. }
$$

Proof. Let $\left\{K_{j}\right\}$ be a monotone divergent sequence of compact sets with $\bigcup K_{j}=M$ such that $M-K_{j}$ is an open half cylinder. Set $p_{j}:=p\left(K_{j}\right)$. Then

$$
\bigcup D_{p_{j}}\left(K_{j}\right)=M \quad \text { and } c\left(D_{p_{j}}\left(K_{j}\right)\right)=2 \pi \chi(M)-2 \pi+\theta_{j} \text {, }
$$

where $\theta_{j}$ is the inner angle of $D_{p_{j}}\left(K_{j}\right)$ at $p_{j}$. Thus,

$$
c(M)=\lim _{j \rightarrow \infty} c\left(D_{p_{j}}\left(K_{j}\right)\right)=2 \pi \chi(M)-2 \pi+\lim _{j \rightarrow \infty} \theta_{j} .
$$

Since $2 \pi \chi(M)-c(M) \geq 2 \pi, \lim _{j \rightarrow \infty} \theta_{j}=0$. Therefore Lemma 2.2 follows from Lemma 2.1 .

Lemma 2.3. For any compact domain $K^{\prime} \subset M$ such that $M-K^{\prime}$ is homeomorphic to an open half cylinder, there exists a compact convex domain $K$ bounded by a simple closed curve such that $K^{\prime} \subset K$.

Proof. Let $\Gamma$ be the set of all simple closed curves freely homotopic to $\partial K^{\prime}$ in $M-\operatorname{Int}\left(K^{\prime}\right)$. Let $\left\{c_{i}\right\}$ be a sequence of elements of $\Gamma$ such that

$$
\lim _{i \rightarrow \infty} L\left(c_{i}\right)=\inf _{c \in \Gamma} L(c) .
$$

For each $c_{i}$ and for an arbitrary fixed point $x_{i}$ on $c_{i}$ there is a $\gamma_{i} \in \Gamma$ such that

$$
L\left(\gamma_{i}\right)=\inf \left\{L(c) ; c \in \Gamma, c \text { passes through } x_{i}\right\} .
$$

If there exists a subsequence $\left\{x_{j}\right\}$ of $\left\{x_{i}\right\}$ such that $\left\{x_{j}\right\}$ converges to some point in $M$, then $\lim \gamma_{j}$ becomes a simple closed curve. The $\lim \gamma_{j}$ bounds a compact convex domain, say, $K$. If $\left\{x_{i}\right\}$ does not contain any convergent subsequence, then neither does $\left\{\gamma_{i}\right\}$ and hence $\gamma_{i}$ is a geodesic loop for all sufficiently large $i$. Let $D_{i}$ be a compact domain bounded by $\gamma_{i}$ and let $\theta_{i}$ be the inner angle of $D_{i}$ at $x_{i}$. The Gauss-Bonnet Theorem implies

$$
c\left(D_{i}\right)=2 \pi \chi(M)-\pi+\theta_{i} .
$$

Since $\bigcup D_{i}=M$,

$$
c(M)=2 \pi \chi(M)-\pi+\lim _{i \rightarrow \infty} \theta_{i},
$$

which contradicts $2 \pi \chi(M)-c(M) \geq 2 \pi$. This completes the proof of Lemma 2.3.

Lemma 2.4. Let $\left\{p_{i}\right\}$ be a divergent sequence of points of $M$ and let $K$ be a compact domain of $M$ such that $M-K$ is homeomorphic to an open half cylinder. Then there exists a number $i(K)$ such that $A_{p_{i}}^{\prime}(K)$ is nonempty for all $i \geq i(K)$.

Proof. Suppose that there exists a divergent sequence $\left\{p_{i}\right\}$ such that all rays emanating from $p_{i}$ intersect $K$. From Lemma 2.3, without loss of generality we 
may assume that $K$ is a convex set and each ray emanating from $p_{i}$ intersects $\operatorname{Int}(K)$. Take a point $p_{i}$ with $p_{i} \in M-K$ and fix it. Assume that $\partial K$ is parametrized positively by arc length relative to $K$. Let $f_{a}: A_{p_{i}} \rightarrow \partial K(a=0,1)$ be the mappings defined by

$$
f_{a}(v):=\exp _{p_{i}} t_{a}\left(\gamma_{v}\right) \quad \text { for } v \in A_{p_{i}} .
$$

Convexity of $K$ implies that there exists a unique minimal subarc $J_{a}^{i}$ of $\partial K$ such that $f_{a}\left(A_{p_{i}}\right) \subset J_{a}^{i}$. All rays emanating from $p_{i}$ pass through points on $J_{1}^{i}$. The endpoints of $J_{1}^{i}$ are denoted by $f_{1}\left(v_{i}\right)$ and $f_{1}\left(w_{i}\right)$ for $v_{i}, w_{i} \in A_{p_{i}}$. Set $\sigma_{i}:=\gamma_{v_{i}}$ and $\tau_{i}:=\gamma_{w_{i}}$. Let $D_{i}^{\prime}$ be a domain in $M-K$ homeomorphic to an open half plane whose boundary consists of $\sigma_{i}\left|\left[t_{1}\left(\sigma_{i}\right), \infty\right), \tau_{i}\right|\left[t_{1}\left(\tau_{i}\right), \infty\right)$ and $\partial K-J_{1}^{i}$. By Lemma $1.3(1)$ we get a monotone divergent sequence $\left\{t_{j}\right\}$ such that each $S_{t_{j}}\left(p_{i}\right)$ is a piecewise smooth simple closed curve and $K \subset$ $B_{t_{1}}\left(p_{i}\right)$. By the choice of $D_{i}^{\prime}$, any ray emanating from $p_{i}$ does not intersect the arc $S_{t_{j}}\left(p_{i}\right) \cap D_{i}^{\prime}$. The same argument as developed in the outline of the proof of Lemma 1.1 implies that there are a cut point $q_{j}$ to $p_{i}$ in $S_{t_{j}}\left(p_{i}\right) \cap D_{i}^{\prime}$ and minimizing geodesic segments $\xi_{j}, \eta_{j}:\left[0, t_{j}\right] \rightarrow M$ joining $p_{i}$ to $q_{j}$ with $\lim \xi_{j}=\sigma_{i}$ and $\lim \eta_{j}=\tau_{i}$. Since $\sigma_{i}$ and $\tau_{i}$ intersect $\operatorname{Int}(K)$, both $\xi_{j}$ and $\eta_{j}$ for each sufficiently large $j$ intersect $\operatorname{Int}(K)$. Let $F_{j}^{i}$ be a disk domain in $M-K$ bounded by $\xi_{j}\left|\left[t_{1}\left(\xi_{j}\right), t_{j}\right], \eta_{j}\right|\left[t_{1}\left(\eta_{j}\right), t_{j}\right]$ and the subarc of $\partial K$ from $\xi_{j}\left(t_{1}\left(\xi_{j}\right)\right)$ to $\eta_{j}\left(t_{1}\left(\eta_{j}\right)\right)$ which is contained entirely in $\partial K-J_{1}^{i}$. Let $\Delta_{i}$ be a disk domain in $M-K$ bounded by $\sigma_{i}\left|\left[0, t_{0}\left(\sigma_{i}\right)\right), \tau_{i}\right|\left[0, t_{0}\left(\tau_{i}\right)\right)$ and the subarc of $\partial K$ joining $\sigma_{i}\left(t_{0}\left(\sigma_{i}\right)\right)$ and $\tau_{i}\left(t_{0}\left(\tau_{i}\right)\right)$. Set $D_{i}:=D_{i}^{\prime}-\operatorname{Cl}\left(\Delta_{i}\right)$ (see Figure 1).

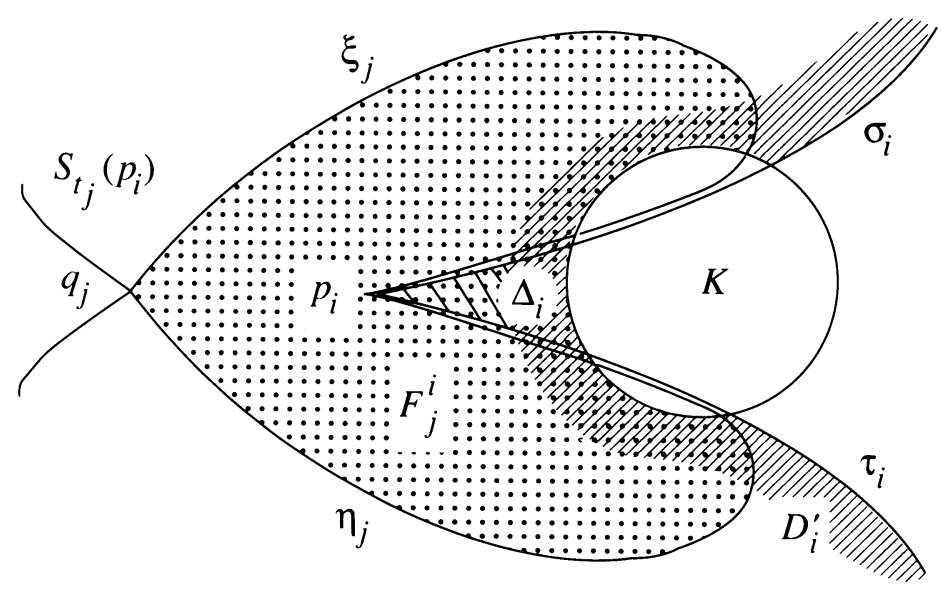

FIGURE 1. 
Since $\bigcup_{j} F_{j}^{i}=D_{i}^{\prime}$, a slight modification of the discussion in the outline of the proof of Lemma 1.1 implies

$$
c\left(D_{i}\right)=\pi-\kappa\left(D_{i}\right) \quad \text { and } \quad c\left(D_{i}^{\prime}\right)=\pi-\kappa\left(D_{i}^{\prime}\right) .
$$

Since $\sigma_{i}$ and $\tau_{i}$ intersect $K$ for all $i$, there are subsequences $\left\{\sigma_{k}\right\}$ and $\left\{\tau_{k}\right\}$ such that $\lim \sigma_{k}=\sigma$ and $\lim \tau_{k}=\tau$ for some straight lines $\sigma$ and $\tau$. Then either $\sigma=\tau$ or $\sigma \cap \tau=\varnothing$. Since $K$ contains finitely many handles, there are two cases for the configuration of four points $\sigma\left(t_{0}(\sigma)\right), \sigma\left(t_{1}(\sigma)\right), \tau\left(t_{0}(\tau)\right)$, $\tau\left(t_{1}(\tau)\right)$ on $\partial K$.

Case 1. The four points $\sigma\left(t_{0}(\sigma)\right), \tau\left(t_{0}(\tau)\right), \tau\left(t_{1}(\tau)\right), \sigma\left(t_{1}(\sigma)\right)$ lie on $\partial K$ in this order. Choose disjoint two open half planes $H_{\sigma}$ and $H_{\tau}$ in $M-K$ such that $H_{\sigma}$ (resp. $H_{\tau}$ ) is bounded by $\sigma\left|\left(-\infty, t_{0}(\sigma)\right], \sigma\right|\left[t_{1}(\sigma), \infty\right)$ and a subarc from $\sigma\left(t_{0}(\sigma)\right)$ of $\partial K$ (resp. $\tau\left(\left(-\infty, t_{0}(\tau)\right]\right), \tau\left(\left[t_{1}(\tau), \infty\right)\right)$ and a subarc from $\tau\left(t_{0}(\tau)\right)$ to $\tau\left(t_{1}(\tau)\right)$ of $\left.\partial K\right)$. Since the inner angle of $D_{k}$ at $p_{k}$ tends to $2 \pi$ as $k \rightarrow \infty$ by Lemma 1.2 , we have

$$
\lim \kappa\left(D_{k}\right)=\kappa\left(H_{\sigma} \cup H_{\tau}\right)-\pi .
$$

On the other hand, $c\left(H_{\sigma}\right) \leq-\kappa\left(H_{\sigma}\right)$ and $c\left(H_{\tau}\right) \leq-\kappa\left(H_{\tau}\right)$ by the remark (1.4). Hence

$$
c\left(H_{\sigma} \cup H_{\tau}\right) \leq-\kappa\left(H_{\sigma} \cup H_{\tau}\right) .
$$

For a fixed number $\varepsilon \in(0, \pi / 2)$ choose a compact set $L$ as to satisfy

$$
\int_{M-L} G^{+} d M<\varepsilon \text { and } c\left(\left(H_{\sigma} \cup H_{\tau}\right) \cap L\right)<-\kappa\left(H_{\sigma} \cup H_{\tau}\right)+\varepsilon .
$$

Then, it follows from (2.4.1) and (2.4.3) that

$\pi-\kappa\left(D_{k}\right)=c\left(D_{k}\right)<c\left(D_{k} \cap L\right)+\varepsilon<c\left(\left(H_{\sigma} \cup H_{\tau}\right) \cap L\right)+2 \varepsilon<-\kappa\left(H_{\sigma} \cup H_{\tau}\right)+3 \varepsilon$

for sufficiently large $k$. Since $\varepsilon<\pi / 2$, this contradicts (2.4.2).

Note that the above arguments imply that $\sigma=\tau$ does not occur.

Case 2. The four points $\sigma\left(t_{0}(\sigma)\right), \tau\left(t_{1}(\tau)\right), \sigma\left(t_{1}(\sigma)\right), \tau\left(t_{0}(\tau)\right)$ lie on $\partial K$ in this order. Let $H_{\sigma}$ be the open half plane in $M-K$ bounded by $\sigma \mid\left(-\infty, t_{0}(\sigma)\right]$, $\sigma \mid\left[t_{1}(\sigma), \infty\right)$ and by a subarc of $\partial K$ such that $\tau\left(\left(-\infty, t_{0}(\tau)\right)\right) \subset H_{\sigma}$ and let $H_{\tau}$ be defined similarly. Then, it follows from (1.3) and (1.4) that $c\left(H_{\tau}\right) \leq-\kappa\left(H_{\tau}\right)$ and $c\left(H_{\sigma}-H_{\tau}\right) \leq \pi-\kappa\left(H_{\sigma}-H_{\tau}\right)$. Hence,

$$
c\left(H_{\sigma} \cup H_{\tau}\right) \leq-\kappa\left(H_{\sigma} \cup H_{\tau}\right) .
$$

Let $\varepsilon \in(0, \pi / 4)$ be a fixed number, and let $L$ be a compact domain such that

$$
\int_{M-L} G^{+} d M<\varepsilon
$$

Then, it follows from (2.4.1) that

$$
\pi-\kappa\left(D_{k}^{\prime}\right)=c\left(D_{k}^{\prime}\right)<c\left(D_{k}^{\prime} \cap L\right)+\varepsilon<c\left(\left(H_{\sigma} \cup H_{\tau}\right) \cap L\right)+2 \varepsilon
$$




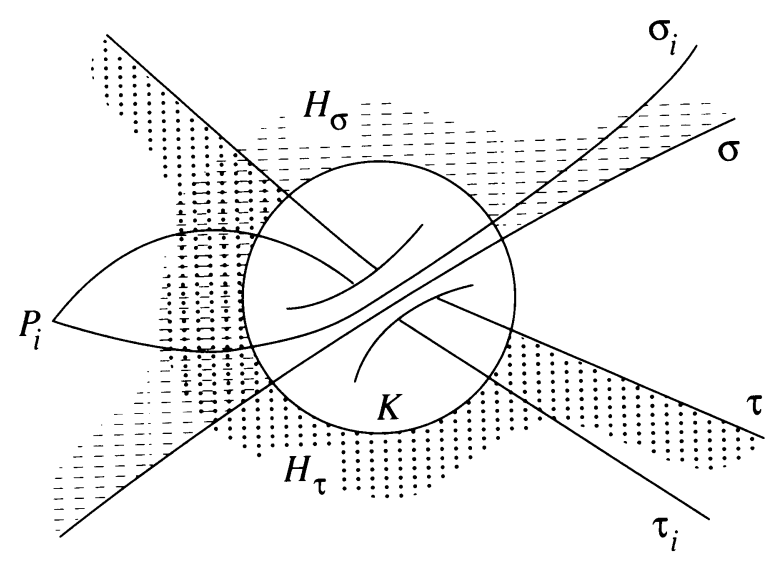

Figure 2.

for sufficiently large $k$. This means that $c\left(H_{\sigma} \cup H_{\tau}\right)$ is finite. Therefore,

$$
\pi-\kappa\left(D_{k}^{\prime}\right)<c\left(H_{\sigma} \cup H_{\tau}\right)+3 \varepsilon \leq-\kappa\left(H_{\sigma} \cup H_{\tau}\right)+3 \varepsilon .
$$

for all sufficiently large $k$. Moreover, since $\sigma_{k}, \tau_{k}$ tend to $\sigma, \tau$,

$$
\lim \kappa\left(D_{k}^{\prime}\right)=\kappa\left(H_{\sigma} \cup H_{\tau}\right) .
$$

This contradicts $\varepsilon<\pi / 4$. This completes the proof of Lemma 2.4 .

Proof of Theorem A. Let $\left\{p_{i}\right\}$ be an arbitrary divergent sequence of points of $M$ and let $K$ be a compact convex domain as obtained in Lemma 2.3. In view of Lemmas 2.2 and 2.4 we may assume that $A_{p_{i}}(K)$ and $A_{p_{i}}^{\prime}(K)$ are nonempty for all $i$. Let $\gamma_{i}, \rho_{i}$ be two rays bounding $D_{p_{i}}(K)$. If $\left\{p_{i}\right\}$ contains a subsequence $\left\{p_{j}\right\}$ such that both $\left\{\gamma_{j}\right\}$ and $\left\{\rho_{j}\right\}$ converge to straight lines, then it follows from Lemmas 1.2 and 2.1 that

$$
\lim \operatorname{meas}\left(A_{p_{j}}\right)=2 \pi \text {. }
$$

Now assume that $\left\{\gamma_{i}\right\}$ does not contain any convergent subsequence. For any $i$, let $\sigma_{i}, \tau_{i}$ be the rays as defined in the proof of Lemma 2.4 and let $D_{i}$ be the open half plane bounded by $\gamma_{i}, \sigma_{i}\left|\left[0, t_{0}\left(\sigma_{i}\right)\right), \sigma_{i}\right|\left[t_{1}\left(\sigma_{i}\right), \infty\right)$ and by a subarc of $\partial K$ such that $D_{i} \subset D_{p_{i}}(K)-K$. Without loss of generality we may assume that $D_{i}$ does not contain $\tau_{i}\left(\left[t_{1}\left(\tau_{i}\right), \infty\right)\right)$. Then there are no rays emanating from $p_{i}$ in $D_{i}$. By a discussion similar to the proof of Lemma 2.4, it follows that

$$
c\left(D_{i}\right)=\pi-\kappa\left(D_{i}\right) .
$$

Since each $\sigma_{i}$ intersects $K$, there is a subsequence $\left\{\sigma_{j}\right\}$ of $\left\{\sigma_{i}\right\}$ converging to some straight line $\sigma$ intersecting $K$. Let $H$ be the open half plane in $M-K$ bounded by $\sigma\left|\left(-\infty, t_{0}(\sigma)\right], \sigma\right|\left[t_{1}(\sigma), \infty\right)$ and a subarc from $\sigma\left(t_{1}(\sigma)\right)$ to $\sigma\left(t_{0}(\sigma)\right)$ of $\partial K$. Since $\left\{\gamma_{j}\right\}$ does not contain any convergent subsequence, $D_{j}$ tends to $H$. By (1.4),

$$
c(H) \leq-\kappa(H) .
$$


For any positive number $\varepsilon$, there exists a compact set $L$ such that

$$
\int_{M-L} G^{+} d M<\varepsilon
$$

Then it follows from (2.A.1) and (2.A.3) that

$$
\pi-\kappa\left(D_{j}\right)=c\left(D_{j}\right)<c\left(D_{j} \cap L\right)+\varepsilon<c(H \cap L)+2 \varepsilon
$$

for all sufficiently large $j$. This means that $c(H)$ is finite. Hence, by (2.A.2),

$$
\pi-\kappa\left(D_{j}\right)<c(H \cap L)+2 \varepsilon<c(H)+3 \varepsilon \leq-\kappa(H)+3 \varepsilon
$$

for all sufficiently large $j$. On the other hand, if we denote by $\psi_{j}$ the inner angle of $D_{j}$ at $p_{j}$, then

$$
\lim \left[\kappa\left(D_{j}\right)-\pi+\psi_{j}\right]=\kappa(H) .
$$

Thus $\psi_{j}<4 \varepsilon$ for all sufficiently large $j$. Thus the argument above applies to $\rho_{i}$ and implies that the inner angle between $\rho_{i}$ and $\tau_{i}$ at $p_{i}$ also tends to zero. From Lemma 1.2 the angle between $\sigma_{i}$ and $\tau_{i}$ at $p_{i}$ tends to zero as $i \rightarrow \infty$. This completes the proof of Theorem A.

\section{The CASE When $M$ HAS MORE THAN ONE END}

The aim of this section is to prove Theorem B. We assume that $M$ has $k$ ends and admits total curvature.

Proof of Theorem B. Let $K$ be a compact domain and $U_{i}$ a tube as in $\S 0$. Let $M_{i}$ be a complete open surface with one end such that there exists an isometric embedding $l_{i}: U_{i} \cup K \rightarrow M_{i}$ and $M_{i}-l_{i}\left(U_{i} \cup K\right)$ consists of $k-1$ open disk domains. Then the Gauss-Bonnet Theorem implies

$$
s_{i}(M)=2 \pi \chi\left(M_{i}\right)-c\left(M_{i}\right) .
$$

Now, without loss of generality we may assume that $K$ contains a compact domain $K^{\prime}$ such that $M-K^{\prime}$ is a disjoint union of $k$ tubes and that $d\left(M-K, K^{\prime}\right)$ is greater than the length of $\partial K^{\prime}$. Then each minimizing segment joining two points in $U_{i}$ is contained in $K \cup U_{i}$. For any $p$ in $U_{i}$, set

$$
A_{p}(i):=\left\{v \in A_{p} ; \gamma_{v}([0, \infty)) \subset U_{i} \cup K\right\}
$$

and let $A_{i, p}$ be the set of all unit vectors at $l_{i}(p)$ tangent to rays emanating from $l_{i}(p)$ in $M_{i}$. It follows that the restriction of differential mapping $d l_{i} \mid A_{p}(i): A_{p}(i) \rightarrow A_{i, p}$ is bijective. In particular we have

$$
\operatorname{meas}\left(A_{p}(i)\right)=\operatorname{meas}\left(A_{i, p}\right)
$$

for all $p$ in $U_{i}$. It follows from the construction of $M_{i}$ that $A_{p}(i) \subset A_{p}=$ $A_{p}(i) \cup A_{p}(K)$ and

$$
\operatorname{meas}\left(A_{i, p}\right) \leq \operatorname{meas}\left(A_{p}\right) \leq \operatorname{meas}\left(A_{i, p}\right)+\operatorname{meas}\left(A_{p}(K)\right)
$$


for all $p$ in $U_{i}$. On the other hand, for a divergent sequence $\left\{p_{j}\right\}$ of points in $U_{i}$, Theorem A and Lemma 1.2 imply

$$
\lim _{j \rightarrow \infty} \operatorname{meas}\left(A_{i, p_{j}}\right)=\min \left\{2 \pi \chi\left(M_{i}\right)-c\left(M_{i}\right), 2 \pi\right\}
$$

and

$$
\lim _{j \rightarrow \infty} \operatorname{meas}\left(A_{p_{j}}(K)\right)=0 .
$$

Therefore, by (3.B.1), (3.B.2), (3.B.3), and (3.B.4),

$$
\lim _{j \rightarrow \infty} \operatorname{meas}\left(A_{p_{j}}\right)=\lim _{j \rightarrow \infty} \operatorname{meas}\left(A_{i, p_{j}}\right)=\min \left\{s_{i}(M), 2 \pi\right\} .
$$

This completes the proof of Theorem B.

\section{ACKNOWLEDGMENT}

The author would like to express his thanks to Professor K. Shiohama for his assistance during the preparation of this paper.

\section{REFERENCES}

1. S. Cohn-Vossen, Kürzeste Wege und Totalkrümmung auf Flächen, Composito Math. 2 (1935), 63-133.

2. - Totalkrümmung und geodätische Linien auf einfach zusammenhängenden offenen volständigen Flächenstücken, Recueil Math. Moscow 43 (1936), 139-163.

3. F. Fiala, Le problème isopérimètres sur les surface onvretes à courbure positive, Comment. Math. Helv. 13 (1941), 293-346.

4. P. Hartman, Geodesic parallel coordinates in the large, Amer. J. Math. 86 (1964), 705-727.

5. M. Maeda, On the existence of rays, Sci. Rep. Yokohama Nat. Univ. 26 (1979), 1-4.

6. _ A geometric significance of total curvature on complete open surfaces, in Geometry of Geodesics and Related Topics, Advanced Studies in Pure Math. 3 (1984), 451-458, Kinokuniya, Tokyo, 1984.

7. __ On the total curvature of noncompact Riemannian manifolds II, Yokohama Math. J. 33 (1985), 93-101.

8. T. Oguchi, Total curvature and measure of rays, Proc. Fac. Sci. Tokai Univ. 21 (1986), 1-4.

9. K. Shiga, On a relation between the total curvature and the measure of rays, Tsukuba J. Math. 6 (1982), 41-50.

10. _ $\ldots$ A relation between the total curvature and the measure of rays II, Tôhoku Math. J. 36 (1984), 149-157.

11. K. Shiohama, Cut locus and parallel circles of a closed curve on a Riemannian plane admitting total curvature, Comment. Math. Helv. 60 (1985), 125-138.

12. _. Total curvatures and minimal areas of complete open surfaces, Proc. Amer. Math. Soc. 94 (1985), 310-316.

13. __ An integral formula for the measure of rays on complete open surfaces, J. Differential Geom. 23 (1986), 197-205.

14. K. Shiohama, T. Shioya and M. Tanaka, Mass of rays on complete open surfaces, preprint. JAPAN

Department of Mathematics, Faculty of Science, Kyushu University, Fukuoka 812, 